NASA/CR-1998-208734

ICASE Report No. 98-46

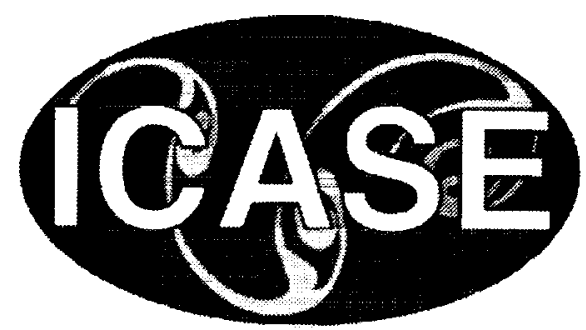

\title{
Vibrational Control of a Nonlinear Elastic Panel
}

\author{
P.L. Chow
}

Wayne State University, Detroit, Michigan

L. Maestrello

NASA Langley Research Center, Hampton, Virginia

Institute for Computer Applications in Science and Engineering NASA Langley Research Center

Hampton, VA

Operated by Universities Space Research Association

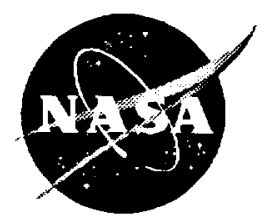

National Aeronautics and

Space Administration

Langley Research Center

Hampton, Virginia 23681-2199

Prepared for Langley Research Center under Contract NAS 1-19480

October 1998 
Available from the following:

NASA Center for AeroSpace Information (CASI)

7121 Standard Drive

Hanover, MD 21076-1320

(301) 621-0390
National Technical Information Service (NTIS)

5285 Port Royal Road

Springfield, VA 22161-2171

(703) $487-4650$ 


\title{
VIBRATIONAL CONTROL OF A NONLINEAR ELASTIC PANEL
}

\author{
P.L. CHOW* AND L. MAESTRELLO ${ }^{\dagger}$
}

\begin{abstract}
The paper is concerned with the stabilization of the nonlinear panel oscillation by an active control. The control is actuated by a combination of additive and parametric vibrational forces. A general method of vibrational control is presented for stabilizing panel vibration satisfying a nonlinear beam equation. To obtain analytical results, a perturbation technique is used in the case of weak nonlinearity. Possible application to the other type of problems is briefly discussed.
\end{abstract}

Key words. vibrational control, stabilization, nonlinear panel oscillation, perturbation technique

Subject classification. Applied and Numerical Mathematics

1. Introduction. The problem under consideration is the stabilization of the nonlinear panel oscillation by an active control with a vibrational actuator. This work was motivated by the recent experimental investigations of the second author (Maestrello $[1,2]$ ), who demonstrated clearly that the vibrational control could be an effective means of stabilizing the boundary-layer flow as well as the panel vibration. This paper will offer a general method of vibrational control and its application to the problem involving a nonlinear elastic panel excited by the periodic wall-pressure fluctuation in a boundary-layer flow.

The general principle of active control, the vibrational control in particular, is to introduce an action which affects a change in the behavior of a dynamical system in a desirable manner. In the boundary layer transition control [1], the periodic heating and cooling of the wall induce a parametric vibration of the fluid viscosity which, in turn, stabilizes the flow. In the case of panel vibration [2], a properly added vibrational force with the same forcing frequency may result in suppressing the subharmonic oscillations (see Section 2). The suppression of subharmonics, of course, has the implication of controlling the chaotic motion.

The main idea of vibrational control stems from the fact that an inverted pendulum can be stabilized at its upper equilibrium position when the lower suspension point executes a rapid vertical vibration (see e.g. [3]). Based on this idea, a general principle of vibrational control was proposed, notably by Meerkov [4], to stabilize the equilibrium points of some finite-dimensional linear systems. Application of this principle to reactor dynamics was done by Bellman et al. [5]. By contrast, in this paper, we will extend this control principle to stabilize the periodic motions of infinite-dimensional systems, instead of equilibrium points in finite dimensions. In addition to the high-frequency parametric vibrational control used in $[4,5]$, a vibrational force with the same forcing frequency will be required. Unlike the usual feedback of feed-forward control, the vibrational control does not need accurate measurement of the system inputs and outputs and can be implemented much more easily, especially for an infinite-dimensional system under consideration.

In this paper we consider the panel vibration which satisfies the initial boundary value problem for the

\footnotetext{
*Department of Mathematics, Wayne State University, Detroit, MI 48202 (email: plchow@math.wayne.edu). This research was supported by the National Aeronautics and Space Administration under NASA Contract No. NAS1-19480 while the author was in residence at the Institute for Computer Applications in Science and Engineering (ICASE), NASA Langley Research Center, Hampton, VA 23681.

${ }^{\dagger}$ Acoustics Division, NASA Langley Research Center, Hampton, VA 23681 (email: Imaestrello@larc.nasa.gov).
} 
nonlinear beam equation [6]:

$$
\left\{\begin{array}{c}
m \partial_{t}^{2} w+c \partial_{t} w-[Q+N(t)] \partial_{x}^{2} w+D \partial_{x}^{4} w=\Delta p(t, x), 0<x<l \\
w(t, 0)=w(t, l)=0, \partial_{x}^{2} w(t, 0)=\partial_{x}^{2} w(t, l)=0 \\
w(0, x)=w_{0}(x), \partial_{t} w(0, x)=w_{1}(x)
\end{array}\right.
$$

Here $w$ denotes the transverse deflection; $\partial_{t}, \partial_{x} \ldots$ are the partial differentiations in $t, x \ldots$; the positive constants $m, c$ and $D$ represent the unit mass, the damping coefficient and the bending stiffness of the panel, respectively. The axial force $Q$ is positive or negative according to the force being tensile or compressive. The large panel deflection introduces an additional tension $N(t)$ given by

$$
N(t)=b \int_{0}^{l}\left|\partial_{x} w(t, x)\right|^{2} d x
$$

where $b$ is an elastic constant. The forcing term $\Delta p$ denotes the pressure difference across the panel surfaces. The homogeneous boundary conditions mean that the panel is simply supported, and the initial data $w_{0}$ and $w_{1}$ are given. Suppose that, without any control, the periodic solution of equation (1.1) excited by the pressure $\Delta p$ is unstable. Our problem is to stabilize the panel oscillation by applying an appropriate control in the form of vibrational forces added to the axial force and the pressure $\Delta p$.

The paper is organized as follows. To illustrate the basic ideas involved, in Section 2, we consider the control of the Duffing equation, for which the response characteristics to a time-harmonic excitation is well known. The feasibility of the vibrational control can be discussed geometrically by referring to the response curves. Since the applicability of vibrational control is not limited to the structure dynamics, in Section 3, a general method of vibrational control for a class of nonlinear evolution equations is presented. For a given unstable periodic solution, the control strategy is to shift the Liapunov exponent $r$ of the vibrational equation to the negative half-line so that the corresponding periodic solution becomes stable. This method is applied to the nonlinear panel vibration problem satisfying equation (1.1). For weak nonlinearity, analytic results are obtained by a perturbation analysis and the case of single-mode excitation is worked out in detail. Finally, in Section 5, some concluding remarks are made and other possible applications such as the flow stabilization problem are mentioned.

2. Control of Duffing's Equation. Before dealing with the nonlinear beam equation (1.1), we consider the Duffing equation

$$
\ddot{y}+\mu \dot{y}+\delta y+\beta y^{3}=F \cos \omega t
$$

where the dot denotes the time derivative, the constants $\mu, \delta, \beta$, and $F$ are assumed to be positive here, and $\omega>0$ is the forcing frequency. For small $F$, by perturbation analysis [7], it is known that equation (2.1) has a periodic solution of the form

$$
y=A \cos (\omega t+\theta)
$$

for some phase shift $\theta$, where the amplitude $A$ is related to the frequency $\omega$ by the response equation [8].

$$
\left[\left(\omega^{2}-\delta\right) A-\frac{3}{4} \beta A^{3}\right]^{2}+\mu \omega^{2} A^{2}=F^{2} .
$$




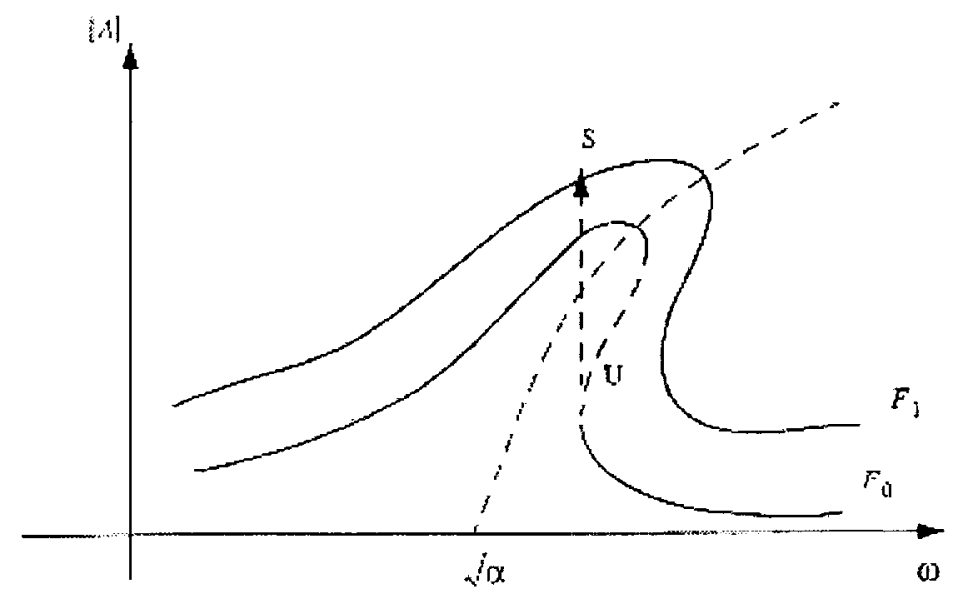

FIG. 2.1. Response curves for harmonic oscillation

By varying the value of $F$, equation (2.3) yields a family of response curves in the $|A|-\omega$ plane. Referring to Fig. 2.1 for $F=F_{0}, F_{1}$, the solid portion of the curve corresponds to the stable regime for the periodic solution (2.2), while the dotted part of the curve (between two points of vertical tangency) renders the solution unstable. With the frequency $\omega$ fixed, point $U$ on the $F_{0}$-curve is unstable, but, by changing $F_{0}$ to $F_{1}$, point $U$ moves up to point $S$ on the $F_{1}$-curve becoming a stable point. Therefore, in this case by adding an in-phase force with the same frequency, an unstable periodic motion can be stabilized. On the other hand, if the forcing amplitude $F$ is large, the system may exhibit a subharmonic response. For example, consider the case of subharmonic response with frequency $\frac{\omega}{3}$. Again, by a perturbation analysis, it is found that equation (2.1) has a subharmonic solution of the form [8]:

$$
y=A \cos \left(\omega t+\theta_{1}\right)+B \cos \left(\frac{\omega}{3} t+\theta_{2}\right)
$$

where $A, B$ and $\theta_{1}, \theta_{2}$ are the corresponding amplitudes and phases, which satisfy some response equations. For the subharmonics, the equation reads

$$
\omega^{2}=9 \alpha+\frac{27}{4} \gamma\left(B^{2}+2 f^{2}\right) \pm\left[\left(\frac{27 \gamma f B}{2}\right)^{2}-\mu^{2}\right]^{\frac{1}{2}}
$$

with $f=9 F / 8$. For $F=F_{0}, F_{1}$ with $F_{1}>F_{0}>0$, the response curves associated with (2.4) are given in Fig. 2.2. Note that for $\omega=\omega_{0}$, point $P$ on the $F_{0}$-curve corresponds to a subharmonics with amplitude $r_{0}$. However, this subharmonics will disappear when $F$ changes from $F_{0}$ to $F_{1}$, since $\omega<\omega_{0}$, and $\omega_{1}$ is the smallest frequency for the existence of a subharmonics at $F=F_{1}$. This may explain qualitatively why a subharmonic vibration can be suppressed in the experimental investigation [2] by an additive periodic force, which has the effect of changing the forcing amplitude $F$.

In contrast with the additive vibrational control, the control can be applied parametrically. For instance, we regard the Duffing equation (2.1) as an approximate equation for an inverted pendulum near the upper equilibrium position $(y=0)$, for which $\alpha=-\delta<0$. Clearly $y=0$ is an unstable equilibrium. If the suspension point vibrates at a high frequency $\nu \gg \omega$, the equation (2.1) should be replaced by [3]

$$
\ddot{z}+\mu \dot{z}+[p(\nu t)-\delta] z+\beta z^{3}=F \cos \omega t,
$$




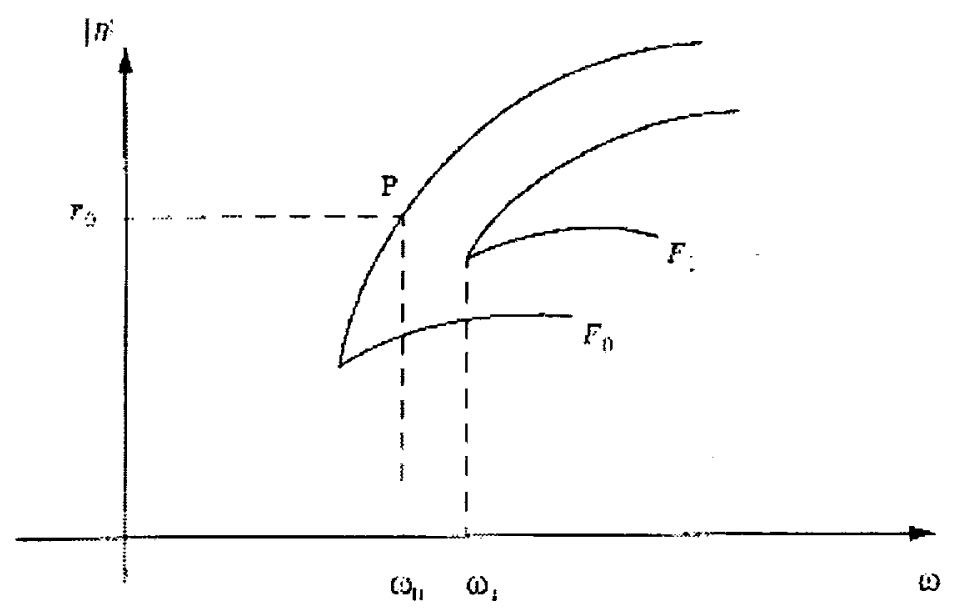

FIG. 2.2. Response curves for subharmonic oscillation

where $p(\tau)=p(\tau+2 \pi)$ is a periodic function. Without the control $p$, a periodic motion about $y=0$ is obviously unstable. However, by the method of averaging [3], equation (2.5) can be closely approximated by the averaged equation (see Section 4):

$$
\ddot{y}+\mu \dot{y}+\left[<p^{2}>-\delta\right] y+\beta y^{3}=F \cos \omega t,
$$

where $\langle p\rangle=0$ and

$$
<p^{n}>=\frac{1}{2 \pi} \int_{0}^{2 \pi} p^{n}(\tau) d \tau, \text { for } n=1,2 .
$$

Therefore, if $\alpha=\left\langle p^{2}>-\delta>0\right.$, the pcriodic motion can now be stabilized as before.

The above examples show the possibility of stabilizing periodic motions by vibrational control. As a generalization we consider the following control problem:

$$
\ddot{z}+\mu \dot{z}+\alpha z+\beta z^{3}=f(\lambda, \omega t)+h(\lambda, \nu t, z)
$$

where $f(\lambda, \tau)=f(\lambda, \tau+2 \pi)$ and $h(\lambda, \tau, z)=h(\lambda, \tau+2 \pi, z)$ are periodic functions; $\lambda$ is a control parameter and $h$ is a certain control function with $h(0, \nu t, y)=0$ and $\omega, \nu$ are the vibration frequencies with $\nu \gg \omega$. The uncontrolled case corresponds to $\lambda=0$ and $h=0$. Of course the equations (2.1) and (2.5) are special cases of (2.7). Suppose that $z=\varphi_{0}(t)$ is an unstable periodic solution of equation (2.7) when $\lambda=0$ and $h=0$. The control objective is to choose the control parameter $\lambda$ and function $h$ so that the corresponding periodic solution $z=\varphi(\lambda, t)$ with $\varphi(0, t)=\varphi_{0}(t)$, becomes asymptotically stable. This means analytically that the variational equation for $y=(z-\varphi)$ from (2.7) has only exponentially decaying solutions. More precisely, if

$$
r(\lambda, \varphi)=\lim _{t \rightarrow \infty} \frac{1}{t} \ln |y(t)|
$$

then we must choose $\lambda$ and $h$ such that $r<0$. Obviously, unlike the optimal control, such a control, if possible, is far from unique. The choice of $\lambda$ and $h$, though guided by physical feasibility, is mostly up to the personal preference. In what follows, this control principle will be generalized to deal with nonlinear partial differential equations. 
3. Stabilization of Nonlinear Evolution Equations. In the theoretical discussion, it is convenient to consider the partial differential equations of interest as a nonlinear evolution equation of the form

$$
\left\{\begin{array}{c}
\frac{d u}{d t}=B(u)+F(\lambda, \omega t)+H(\lambda, \nu t, u), \\
u(0)=h
\end{array}\right.
$$

where $u(t)$ is a vector in some infinite-dimensional vector space $V$ with initial state $h$. The operator $B$ is nonlinear, $F(\lambda, \tau)=F(\lambda, \tau+2 \pi)$ and $H(\lambda, \tau, u)=H(\lambda, \tau+2 \pi, u)$ are periodic with control parameter $\lambda$, being a scalar or vector. The control function $H$ acts parametrically with rapid oscillations so that $\nu>\omega$. When $\lambda=0, H(0, \tau, u)=0$ and the system (3.1) is uncontrolled. We are interested in stabilizing a periodic motion which is unstable at $\lambda=0$. If the equilibrium solution $u_{0}$ of (3.1) at $\lambda=0$ is also unstable, we introduce a parametric vibrational control $H$ to stabilize it as in the case of an inverted pendulum. The effect of $H$ can be examined by the method of averaging [3]. By a change of time from $t$ to $\sigma=t / \varepsilon$ with $\varepsilon=1 / \nu$, the system (3.1) can be approximated by the averaged equation

$$
\left\{\begin{array}{c}
\frac{d u}{d t}=B(u)+F(\lambda, \omega t)+\hat{H}(\lambda, u), \\
u(0)=h,
\end{array}\right.
$$

where

$$
\hat{H}(\lambda, u)=\langle H(\lambda, \nu t, u)\rangle=\frac{1}{2 \pi} \int_{0}^{2 \pi} H(\lambda, \tau, u) d \tau .
$$

The function $H$ should be chosen so that the equilibrium solution of $u_{1}$ of the averaged equation (3.2) becomes stable. Without control $(\lambda=0)$, let $u=\psi_{0}(t)$ be an unstable periodic solution of equation (3.1) near $u_{0}$. In addition to the parametric control $H$, we have modulated the forcing function $F(\lambda, \omega t)$ by tuning the control parameter $\lambda$ so that the corresponding periodic motion satisfying the averaged equation (3.2) is asymptotically stable. To this end let us consider the variational equation of (3.2) for $v=(u-\psi)$ :

$$
\left\{\begin{array}{c}
\frac{d v}{d t}=B_{1}(\psi, v)+H_{1}(\lambda, \psi, v), \\
v(0)=g
\end{array}\right.
$$

where

$$
\begin{aligned}
B_{1}(\psi, v) & =B(v+\psi)-B(\psi), \\
H_{1}(\lambda, \psi, v) & =\hat{H}(\lambda, v+\psi)-\hat{H}(\lambda, \psi),
\end{aligned}
$$

and $g$ is an initial vector in $V$. Let $\|h\|$ denote the magnitude (norm) of vector $h$. The control objective is then to choose function $H$ and parameter $\lambda$ in such a way that the Liapunov exponent $r$ is negative,

$$
r(\lambda, H)=\lim _{t \rightarrow \infty} \frac{1}{t} \ln \|v(t)\|<0,
$$

for all $g$ with $\|g\|<\delta$ with some $\delta>0$. For small $\delta$, the variational equation (3.4) can be linearized to give

$$
\left\{\begin{array}{c}
\frac{d v}{d t}=A(\lambda, t) v \\
v(0)=g
\end{array}\right.
$$

where $A(\lambda, t)$ is a linear operator defined by

$$
A(\lambda, t) v=\left\{B_{u}[\psi(\lambda, t)]+H_{u}[\lambda, \psi(\lambda, t)]\right\} v
$$


and $B_{u}(v)=\frac{\delta B(v)}{\delta v}, H_{u}(\lambda, v)=\frac{\delta \hat{H}(\lambda, v)}{\delta v}$ are the linearized operators of $B$ and $H$ at $v$. Note that $A(\lambda, t)$ is periodic with the same period $T$ as that of $\psi \cdot \lambda$, if $V$ is finite-dimensional and $A(\lambda, t)$ is a matrix, then, by the Floquet theory [7], the solution of equation (3.6) can be expressed as

$$
v(t)=P(t) e^{t R} g
$$

where $P(t)=P(t+T)$ is a periodic matrix, and $R$ is a constant matrix. The smallest real part of the eigenvalues of $R$ yields the Liapunov exponent $r$. Of course the representation (3.4) holds for any finitedimensional approximation of equation (3.4). Unfortunately, even the periodic function $\psi$ is known, the analytical computation of the Liapunov exponent $r$ through either (3.5) or (3.8) is impossible without simplifying assumptions. For example, for small amplitude vibration, the nonlinearity is weak so that the perturbation method and an eigenfunction expansion can be applied. This procedure will be illustrated in the application to the panel vibration problem.

4. Vibrational Control of Elastic Panel. By redefining the constants in the nonlinear beam equation (1.1) under a vibrational control, it yields

$$
\partial_{t}^{2} w+\mu \partial_{t} w-\left(\alpha+\beta\left\|\partial_{x} w\right\|^{2}\right) \partial_{x} w+\gamma \partial_{x}^{4} w=p(\lambda, \omega t, x)+h(\lambda, \nu t, x, w)
$$

where the initial-boundary conditions are omitted, and

$$
\begin{gathered}
\left\|\partial_{x} w\right\|^{2}=\int_{o}^{l}\left|\partial_{x} w\right|^{2} d x \\
p(\lambda, \omega t, x)=\Delta p(\omega t, x)+p_{1}(\lambda, \omega t, x)
\end{gathered}
$$

$p_{1}$ and $h$ are the additive and parametric control forces with frequencies $\omega \gg \nu$. The physical constants $\mu, \beta, \gamma$ are positive, while $\alpha$ is positive or negative depending on the axial force being tensile or compressive. Without control, we assume that, at $\lambda=0, p_{1}(0, x, \tau)=h(0, \sigma, x, w)=0$. To be specific, we choose the parametric control to be a vibrational axial force of the form

$$
h(\lambda, \nu t, x, w)=\dot{q}(\lambda, \nu t) \partial_{x}^{2} w
$$

where

$$
\dot{q}=\partial_{t} q(\lambda, \nu t)
$$

with

$$
<q>=<\dot{q}>=0
$$

Now let $u_{1}=w$ and $u_{2}$ defined by

$$
\dot{u}_{1}=u_{2}+q(\lambda, \nu t) \partial_{x}^{2} u_{1}
$$

Then equations (4.1) and (4.6) yield

$$
\begin{aligned}
\dot{u}_{2}= & -\left\{\mu\left(u_{2}+q \partial_{x}^{2} u_{1}\right)-\left(\alpha+\beta\left\|\partial_{x} u_{1}\right\|^{2}\right) \partial_{x}^{2} u_{1}+\gamma \partial_{x}^{4} u_{1}\right\} \\
& -q\left(\partial_{x}^{2} u_{2}-q \partial_{x}^{4} u_{1}\right)+p(\lambda, \omega t, x)
\end{aligned}
$$


We set

$$
u=\left[\begin{array}{l}
u_{1} \\
u_{2}
\end{array}\right]
$$

and rewrite the equations (4.6) and (4.7) in the form (3.1):

$$
\frac{d u}{d t}=B(u)+F(\lambda, \omega t)+H(\lambda, \omega t, u)
$$

where

$$
\begin{gathered}
B(u)=\left[\begin{array}{c}
u_{2} \\
-\left\{\mu u_{2}-\left(\alpha+\beta\left\|\partial_{x} u_{1}\right\|^{2}\right) \partial_{x}^{2} u_{1}+\gamma \partial_{x}^{4} u_{1}\right\}
\end{array}\right] \\
F(\lambda, \omega t)=\left[\begin{array}{c}
0 \\
p(\lambda, \omega t, \cdot)
\end{array}\right]
\end{gathered}
$$

and

$$
H(\lambda, \nu t, u)=\left[\begin{array}{c}
q \partial_{x}^{2} u_{1} \\
-\mu q \partial_{x}^{2} u_{1}-q^{2} \partial_{x}^{4} u_{1}-q \partial_{x}^{2} u_{2}
\end{array}\right]
$$

In view of equations (4.5) and (4.9) (4.11), by taking the time-average of equation (4.8) in $\sigma=\nu t$ with $\tau=\omega t$ fixed, we get

$$
\frac{d u}{d t}=B(u)+F(\lambda, \omega t)+\hat{H}(\lambda, u)
$$

where

$$
\hat{H}(\lambda, u)=\left[\begin{array}{c}
0 \\
-<q^{2}>\partial_{x}^{4} u_{1}
\end{array}\right]
$$

We note that the average equation (4.12) yields a scalar equation for $w=u_{1}$ as follows,

$$
\partial_{t}^{2} w+\mu \partial_{t}^{2} w-\left(\alpha+\beta\left\|\partial_{x} w\right\|^{2}\right) \partial_{x}^{2} w+\left(\gamma+<q^{2}>\right) w=p(\lambda, \omega t, x)
$$

which shows that the high-frequency axial vibrational force $q(\lambda, \nu t)$ has the effect of increasing the bending stiffness $\gamma$ by the magnitude of $\left\langle q^{2}\right\rangle$. Thus it stabilizes the system statically in general. Now let

$$
u_{0}=\psi_{0}(t)=\left[\begin{array}{c}
\psi_{01}(t) \\
\psi_{02}(t)
\end{array}\right]
$$

be an unstable periodic solution of equation (4.8) when $\lambda=0(H=0)$, and let

$$
u=\psi(\lambda, t)=\left[\begin{array}{l}
\psi_{1}(\lambda, t) \\
\psi_{2}(\lambda, t)
\end{array}\right]
$$

be a periodic solution of equation (4.14) with $\psi(0, t)=\psi_{0}(t)$. Define $v=(u-\psi)$ so that $v$ satisfies the variational equation (3.4). Here it can be written in the form

$$
\frac{d v}{d t}=A(\lambda, t) v+\beta G(\lambda, t, v),
$$


where $A$ is a periodic linear operator and $G$ is a nonlinear mapping defined as

$$
A v=\left[\begin{array}{c}
v_{2} \\
f_{1}
\end{array}\right], G=-\left[\begin{array}{c}
0 \\
f_{2}
\end{array}\right],
$$

and

$$
\begin{aligned}
& f_{1}=-\mu v_{2}+\left(\alpha+<q^{2}>+\beta\left\|\partial_{x} \psi_{1}\right\|^{2}\right) \partial_{x}^{2} v_{1}+2 \beta\left(\partial_{x} \psi_{1}, \partial_{x} v_{1}\right) \partial_{x}^{2} \psi_{1}-\gamma \partial_{x}^{4} v_{1} \\
& f_{2}=\left\|\partial_{x} v_{1}\right\|^{2} \partial_{x}^{2} \psi_{1}+2\left(\partial_{x} \psi_{1}, \partial_{x} v_{1}\right) \partial_{x^{1}}^{2}+\left\|\partial_{x} v_{1}\right\|^{2} \partial_{x}^{2} v_{1}
\end{aligned}
$$

with the inner product notation

$$
(g, h)=\int_{0}^{l} g(x) h(x) d x .
$$

When the nonlinear term $G$ is dropped, equation (4.15) yields a generalized Hill's equation, a linear partial differential equation with periodic coefficient:

$$
\frac{d v}{d t}=A(\lambda, t) v
$$

For computational purposes, introduce a complete set of orthonormal functions $\left\{e_{n}\right\}$, which may be the eigenfunctions associated with the linearized problem, or $e_{n}(x)=\sqrt{\frac{2}{l}} \sin \frac{n \pi}{l} x, n=1,2, \ldots$ By the expansion of the solutions of (4.12) and (4.15) into terms of $e_{n}^{\prime} s$ as follows,

$$
u=\sum_{n=1}^{\infty} u_{n}(t) e_{n} ; v=\sum_{n=1}^{\infty} v_{n}(t) e_{n},
$$

their coefficients satisfy the infinite systems of coupled ordinary differential equations of the form:

$$
\begin{gathered}
\frac{d u_{i}}{d t}=B_{i}\left(u_{1}, \ldots, u_{n}, \ldots\right)+F_{i}(\lambda, t)+\hat{H}_{i}\left(\lambda, u_{1}, \ldots, u_{n}, \ldots\right), \\
\frac{d v_{i}}{d t}=\sum_{n=1}^{\infty} a_{i j}(\lambda, t) v_{j}, i=1,2, \ldots, n, \ldots,
\end{gathered}
$$

where $a_{i j}=\left(A e_{i}, e_{j}\right)$. The above systems can only be solved numerically for truncated systems of low dimensions. As mentioned before, if the nonlinear effect is weak, we can apply the perturbation analysis to approximate solutions analytically. To this end let us assume that the damping coefficient and the forcing amplitude are small. By proper scaling with respect to a small parameter $\varepsilon>0$, the equation (4.14) is rewritten as

$$
\partial_{t}^{2} w+\varepsilon \mu \partial_{t} w-\left(\alpha+\varepsilon \beta\left\|\partial_{x} w\right\|^{2}\right) \partial_{x}^{2} w+\gamma \partial_{x}^{4} w=\varepsilon p(\lambda, \omega t, x)
$$

for which we assume $\alpha>0$ and set $\left\langle q^{2}\right\rangle=0$ for simplicity. It remains to study the problem of additive control. To illustrate the perturbation procedure, we will analyze the case of single-mode excitation in some detail.

Let us consider the case of nth mode harmonic excitation in (4.17):

$$
p(\lambda, \omega t, x)=F(\lambda) \sin \frac{n \pi}{l} x \cos \omega t, n=1,2 \ldots
$$

where the control parameter $\lambda$ modulates the forcing amplitude $F$ and $F_{0}=F(0)$ is the uncontrolled amplitude. Then the equation (4.17) admits a single-mode solution

$$
w=z_{n}(t) \sin \frac{n \pi}{l} x
$$




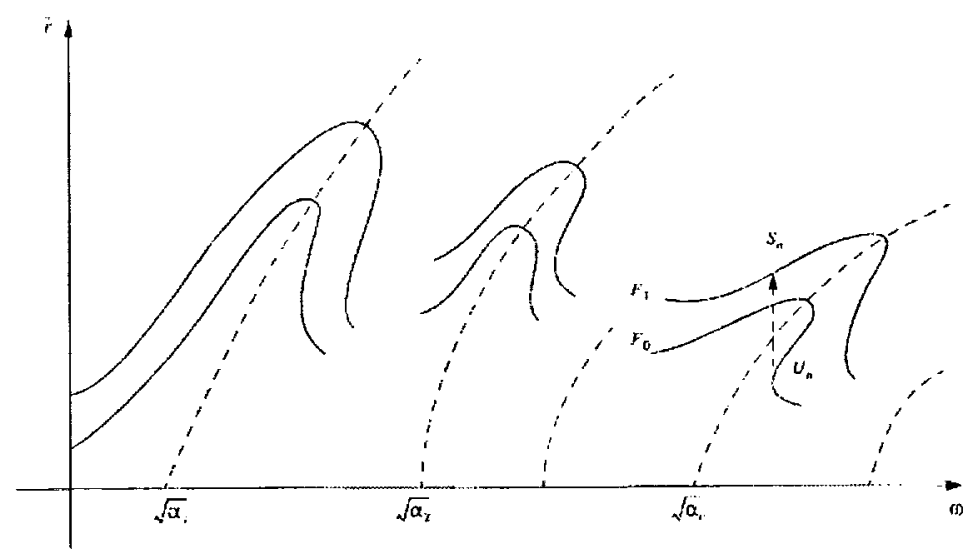

FIG. 4.1. Response curves for different modes of excitations

and $z_{n}$ satisfies the Duffing equation:

$$
\ddot{z}_{n}+\varepsilon \mu \dot{z}_{n}+\alpha_{n} z_{n}+\varepsilon \beta_{n} z_{n}^{3}=\varepsilon F(\lambda) \cos \omega t
$$

where

$$
\begin{aligned}
& \alpha_{n}=\left[\alpha+\gamma\left(\frac{n \pi}{l}\right)^{2}\right]\left(\frac{n \pi}{l}\right)^{2}, \\
& \beta_{n}=\frac{\beta l}{2}\left(\frac{n \pi}{l}\right)^{4} .
\end{aligned}
$$

The perturbation analysis of Duffing's equation has been discussed by many authors (see e.g. [3, 7]). Here we adopt the method of averaging by letting

$$
z_{n}=y_{1}(t) \sin \omega t+y_{2}(t) \cos \omega t
$$

with

$$
\dot{y}_{1} \sin \omega t+\dot{y}_{2} \cos \omega t=0
$$

which are substituted into $(4.20)$ to give

$$
\left\{\begin{array}{c}
\dot{y}_{1}=\frac{1}{2 \omega}\left[\delta_{n} y_{2}-\frac{3}{4} \beta_{n}|y|^{2} y_{2}+\mu w y_{1}+F(\lambda)\right], \\
\dot{y}_{2}=\frac{-1}{2 \omega}\left[\delta_{n} y_{1}-\frac{3}{4} \beta_{n}|y|^{2} y_{1}+\mu w y_{2}\right]
\end{array}\right.
$$

with $\delta_{n}=\left(w^{2}-\alpha_{n}\right)$ and $|y|^{2}=y_{1}^{2}+y_{2}^{2}$. In the polar form, $y_{1}=r \sin \varphi, y_{2}=r \cos \varphi$, this equation becomes

$$
\left\{\begin{array}{l}
\dot{r}=\frac{1}{2 \omega} R(r, \varphi, \omega) \\
\dot{\varphi}=\frac{1}{2 \omega} \Phi(r, \varphi, \omega)
\end{array}\right.
$$

where

$$
\left\{\begin{array}{c}
R=\mu \omega r-F \sin \varphi \\
\Phi=\delta_{n}-\frac{3}{4} \beta_{n} r^{2}+\frac{F}{r} \cos \varphi
\end{array}\right.
$$

The solution (4.21) can be written as

$$
z_{n}=r(t) \cos [\omega t+\varphi(t)]
$$


Therefore, for $z_{n}$ being periodic with frequency $\omega, r$ and $\varphi$ must be constants, which correspond to the cquilibrium point $(\tilde{r}, \tilde{\varphi})$ of equation (4.23) satisfying

$$
\left\{\begin{array}{c}
R(\tilde{r}, \tilde{\varphi}, \omega)=0 \\
\Phi(\tilde{r}, \tilde{\varphi}, \omega)=0
\end{array}\right.
$$

By taking equation (4.24) into account, the above equation can be solved approximately to give

$$
\left(\delta_{n} \tilde{r}-\frac{3}{4} \beta_{n} \tilde{r}^{3}\right)^{2}+\mu \tilde{r}^{2} \omega^{2}=F^{2}(\lambda)
$$

which, by a change of notation, agrees with the response relation (2.3). Therefore for each $n$, the response curves are shown in Fig. 2.1. Schematically, for $n=1,2, \ldots$, the response curves are plotted in Fig. 4.1. Geometrically the control strategy is to steer an unstable point $U_{n}$ on the $F_{o}$-curve to a stable point $S_{n}$ on the $F_{1}$-curve. Analytically the stability of a periodic solution is now reduced to that of an equilibrium point, which can be checked more easily. To do so we form the first variational equation of $(4.23)$ about $(\tilde{r}, \tilde{\varphi})=$

$$
\left\{\begin{array}{l}
\dot{\rho}=\frac{1}{2 \omega}\left(\tilde{R}_{r} \rho+\tilde{R}_{r} \tilde{\Phi}_{\varphi}\right), \\
\dot{\theta}=\frac{1}{2 \omega}\left(\tilde{\Phi}_{r} \rho+\tilde{R}_{\varphi} \tilde{\Phi}_{r}\right),
\end{array}\right.
$$

where $\tilde{R}_{r}=\partial_{r} R(\tilde{r}, \tilde{\varphi}, \omega), \tilde{R}_{\varphi}=\partial_{\varphi} R(\tilde{r}, \tilde{\varphi}, \omega)$ and so on. Let $\eta(\lambda)$ denote an eigenvalue of the coefficient matrix of (4.28). It can be readily verified that, by making use of (4.24) and (4.27), if

$$
D(\lambda)=\left(\tilde{R}_{r} \tilde{\Phi}_{\varphi}-\tilde{R}_{\varphi} \tilde{\Phi}_{r}\right)>0
$$

then $\operatorname{Re} \eta(\lambda)<0$ so that the steady state $(\tilde{r}, \tilde{\varphi})$ is stable. This is of course the stability condition for the associated periodic solution. After computing the partial derivatives in (4.29), it yields

$$
D(\lambda)=\left(\frac{3}{4} \beta_{n} \tilde{r}^{2}-\delta_{n}\right)\left(\frac{9}{4} \beta_{n} \tilde{r}^{2}-\delta_{n}\right)+\mu^{2} \omega^{2}>0,
$$

with $\delta_{n}=\left(\omega^{2}-\alpha_{n}\right)$. The above inequality determines the stability regime $S$ in the $\tilde{r}-\omega$ plane. In view of (4.27), $\tilde{r}(\lambda)$ depends on the control parameter $\lambda$, which will take an unstable point into the stable regime $S$. Note that from (4.30), we can get a simple sufficient stability condition:

$$
\omega^{2}<\alpha_{n}+\frac{3}{4} \beta_{n} \tilde{r}^{2} \lambda
$$

or

$$
\omega^{2}>\alpha_{n}+\frac{9}{4} \beta_{n} \tilde{r}^{2} \lambda
$$

The above inequalities give rise to stable (shaded) sub-regions as shown in Fig. 4.2.

In general, all modes are excited by periodic pressure fluctuations. For instance, consider the harmonic forcing (4.18) with a general spatially dependent amplitude

$$
p(\lambda, \omega t, x)=F(\lambda, x) \cos \omega t .
$$

If the axial load is compressive $(\alpha<0)$ and slightly exceeds the lowest buckling load $\gamma\left(\frac{\pi}{l}\right)^{4}$, the parametric control $q(\nu t)$ can still be used to stabilize the system statically by choosing $\left.\left\langle q^{2}\right\rangle\right\rangle|\alpha|$. So we remain to consider equation (4.17) by assuming $\alpha>0$ there. To apply the above perturbation procedure, we need to 


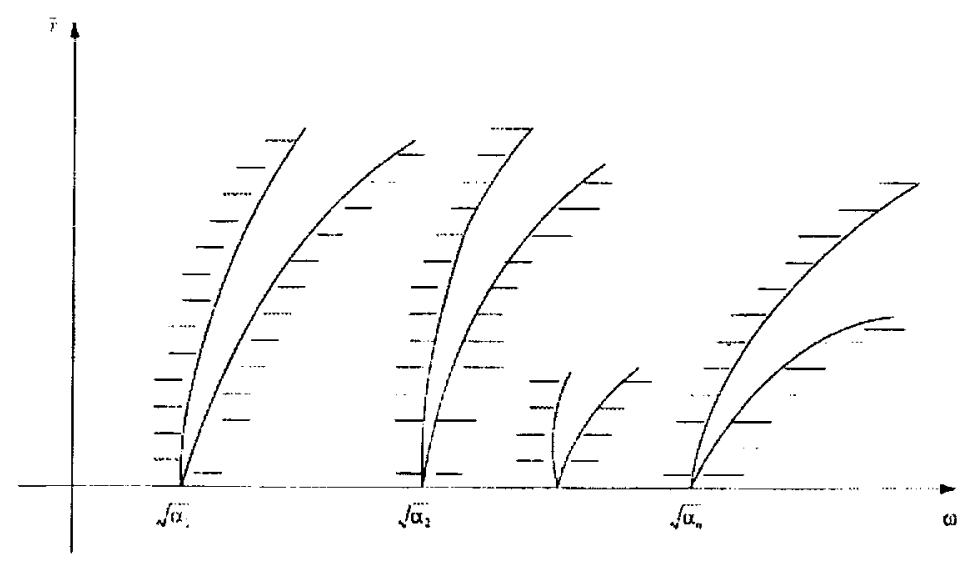

FIG. 4.2. Stability regions (shaded) for different modes of excitations

expand $w$ in (4.17) and $F$ in (4.32) into infinite series with respect to the modal function $e_{n}(x)=\sqrt{\frac{2}{l}} \sin \frac{n \pi}{l} x$, for $n=1,2, \ldots$ The resulting infinite system of coupled nonlinear differential equations for the coefficient functions can then be treated by a perturbation analysis. Such a procedure developed previously for nonlinear wave equations by one of us (Chow [9]) can be applied here. However, unlike the single mode situation, simple stability conditions such as (4.30) or (4.31) are no longer attainable. Though it is possible to study the stability régime numerically after a finite-mode approximation, this has not yct been done.

5. Concluding Remarks. In the paper we present a general method of vibrational control for a certain class of nonlinear evolution equations with a particular reference to the nonlinear beam equation arising from the panel structure dynamics. The control consists of a high frequency parametric vibration and the forcing amplitude modulation. The high-frequency control is to affect a change in system parameter for static stability, while the additive control of the excitation force, if needed, is to stabilize an unstable periodic motion. In application to the panel structure, we show that, for a periodically excited panel near a buckled state, a high frequency oscillatory axial force can keep the system in the state of periodic motion, which can then be stabilized by an additive force modulation. The reason that we only control the force amplitude, instead of both the amplitude and phase is that the additive control is the most effective when it is in phase or out of phase with the excitation force. For a small forcing amplitude, a perturbation technique can be used to reduce the stabilization of a periodic motion to that of an equilibrium point, the latter of which is much simpler to analyze. In the case of a single-modal approximation, the stabilization problem can be studied numerically but has not yet been treated. The vibrational control principle described in this paper can also be applied to other problems such as the flow stability control. Here the nonlinear evolution equation is given by the Navier-Stokes equation. For a slightly unstable flow, the perturbation analysis by Keller and Kogelman [10] can be employed to deal with the flow stabilization by vibrational control. 


\section{REFERENCES}

[1] Maestrello, L., Active transition fixing and control of the boundary layer in air, AIAA Journal 24 (1986), pp. 1577-1581.

[2] Maestrello, L., Active control of nonlinear vibration of a panel structure, Journal of Acoustic Society of America 93 (1993), p. 2252.

[3] Bogoliubov, N.N. and Mitropolskir, Y.A., Asymptotic Methods in the Theory of Nonlinear Oscillations, Gordon and Breach, New York, 1961.

[4] MeErkov, S.M., Principle of vibrational control: Theory and applications, IEEE Transactions on Automatic Control AC-25 (1980), pp. 755-762.

[5] Bellman, R., Bentsman, J., and Meerkov, S.M., Vibrational control of systems with Arhenius dynamics, Journal of Mathematical Analysis and Applications 91 (1993), pp. 152-191.

[6] Dowell, E.H., Aeroelasticity of Plates and Shells, Noordhoff, Leyton, The Netherlands, 1975.

[7] Hale, J.K., Ordinary Differential Equations, Wiley-Interscience, New York, 1969.

[8] STOKER, J.J., Nonlinear Vibrations in Mechanical and Electrical Systems, Wiley-Interscience, New York, 1965.

[9] CHow, P.L., Asymptotic solutions of inhomogeneous initial boundary value problems for weakly nonlinear partial differential equations, SIAM Journal of Applied Mathematics 22 (1972), pp. 629-647.

[10] Keller, J.B. ANd Kogelman, S., Asymptotic solutions of initial value problems for nonlinear partial differential equations, SIAM Journal of Applied Mathematics 18 (1970), pp. 748-758. 
. 


\begin{tabular}{|c|c|c|c|}
\hline \multicolumn{3}{|c|}{ REPORT DOCUMENTATION PAGE } & $\begin{array}{l}\text { Form Approved } \\
\text { OMB No. 0704-0188 }\end{array}$ \\
\hline \multicolumn{4}{|c|}{$\begin{array}{l}\text { Public reporting burden for this collection of information is estimated to average } 1 \text { hour per response, including the time for reviewing instructions, searching existing data sources, } \\
\text { gathering and maintaining the data needed, and completing and reviewing the collection of information. Send comments regarding this burden estimate or any other aspect of this } \\
\text { collection of information, including suggestions for reducing this burden, to Washington Headquarters Services, Directorate for Information Operations and Reports, } 1215 \text { Jefferson } \\
\text { Davis Highway, Suite 1204, Arlington, VA 22202-4302, and to the Office of Management and Budget, Paperwork Reduction Project (0704-0188). Washington. DC 20503. }\end{array}$} \\
\hline 1. AGENCY USE ONLY(Leave blank) & $\begin{array}{l}\text { 2. REPORT DATE } \\
\text { October } 1998\end{array}$ & \multicolumn{2}{|c|}{$\begin{array}{l}\text { 3. REPORT TYPE AND DATES COVERED } \\
\text { Contractor Report }\end{array}$} \\
\hline \multicolumn{3}{|c|}{$\begin{array}{l}\text { 4. TITLE AND SUBTITLE } \\
\text { Vibrational Control of a Nonlinear Elastic Panel }\end{array}$} & \multirow[t]{2}{*}{$\begin{array}{l}\text { 5. FUNDING NUMBERS } \\
\text { C NAS1-19480 } \\
\text { WU } 505-90-52-01\end{array}$} \\
\hline \multicolumn{3}{|l|}{$\begin{array}{l}\text { 6. AUTHOR(S) } \\
\text { P.L. Chow } \\
\text { L. Maestrello }\end{array}$} & \\
\hline \multicolumn{3}{|c|}{$\begin{array}{l}\text { 7. PERFORMING ORGANIZATION NAME(S) AND ADDRESS(ES) } \\
\text { Institute for Computer Applications in Science and Engineering } \\
\text { Mail Stop 403, NASA Langley Research Center } \\
\text { Hampton, VA 23681-2199 }\end{array}$} & $\begin{array}{l}\text { 8. PERFORMING ORGANIZATION } \\
\text { REPORT NUMBER } \\
\text { ICASE Report No. } 98-46\end{array}$ \\
\hline \multicolumn{3}{|c|}{$\begin{array}{l}\text { 9. SPONSORING/MONITORING AGENCY NAME(S) AND ADDRESS(ES) } \\
\text { National Aeronautics and Space Administration } \\
\text { Langley Research Center } \\
\text { Hampton, VA 23681-2199 }\end{array}$} & $\begin{array}{l}\text { 10. SPONSORING/MONITORING } \\
\text { AGENCY REPORT NUMBER } \\
\text { NASA/CR-1998-208734 } \\
\text { ICASE Report No. } 98-46\end{array}$ \\
\hline \multicolumn{4}{|c|}{$\begin{array}{l}\text { 11. SUPPLEMENTARY NOTES } \\
\text { Langley Technical Monitor: Dennis M. Bushnell } \\
\text { Final Report } \\
\text { To be submitted to the Journal of the Acoustic Society of America }\end{array}$} \\
\hline \multicolumn{2}{|c|}{$\begin{array}{l}\text { 12a. DISTRIBUTION/AVAILABILITY STATEMENT } \\
\text { Unclassified-Unlimited } \\
\text { Subject Category } 34 \\
\text { Distribution: Nonstandard } \\
\text { Availability: NASA-CASI (301)621-0390 }\end{array}$} & & 12b. DISTRIBUTION CODE \\
\hline \multicolumn{4}{|c|}{$\begin{array}{l}\text { 13. ABSTRACT (Maximum } 200 \text { words) } \\
\text { The paper is concerned with the stabilization of the nonlinear panel oscillation by an active control. The control is } \\
\text { actuated by a combination of additive and parametric vibrational forces. A general method of vibrational control } \\
\text { is presented for stabilizing panel vibration satisfying a nonlinear beam equation. To obtain analytical results, a } \\
\text { perturbation technique is used in the case of weak nonlinearity. Possible application to the other type of problems } \\
\text { is briefly discussed. }\end{array}$} \\
\hline \multirow[t]{2}{*}{$\begin{array}{l}\text { 14. SUBJECT TERMS } \\
\text { vibrational control; stabilizat }\end{array}$} & ation; nonlinear panel oscillatior & n; perturbation technique & $\begin{array}{l}\text { 15. NUMBER OF PAGES } \\
17\end{array}$ \\
\hline & & 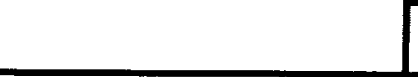 & $\begin{array}{c}\text { 16. PRICE CODE } \\
\mathrm{A} 03\end{array}$ \\
\hline $\begin{array}{l}\text { 17. SECURITY CLASSIFICATION } \\
\text { OF REPORT } \\
\text { Unclassified }\end{array}$ & $\begin{array}{l}\text { 18. SECURITY CLASSIFICATION } \\
\text { OF THIS PAGE } \\
\text { Unclassified }\end{array}$ & $\begin{array}{l}\text { 19. SECURITY CLASSIFICATION } \\
\text { OF ABSTRACT }\end{array}$ & $\begin{array}{l}\text { 20. LIMITATION } \\
\text { OF ABSTRACT }\end{array}$ \\
\hline NSN 7540-01-280-5500 & & & $\begin{array}{l}\text { Standard Form 298(Rev. 2-89) } \\
\text { Prescribed by ANSI Std. Z39-18 } \\
298-102\end{array}$ \\
\hline
\end{tabular}

